\title{
Class of Einstein-Maxwell Dilatons
}

\author{
Tonatiuh Matos*, Darío Núñez ${ }^{\dagger}$ and Hernando Quevedo ${ }^{\dagger}$ \\ * Departamento de Física, Centro de Investigación y Estudios Avanzados del I.P.N. \\ A.P. 14-700, 07000 México D.F. \\ $\dagger$ Instituto de Ciencias Nucleares, UNAM, \\ Circuito Exterior CU, A.P. 70-543, México, D. F. 04510, México.
}

\begin{abstract}
Three different classes of static solutions of the Einstein-Maxwell equations nonminimally coupled to a dilaton field are presented. The solutions are given in general in terms of two arbitrary harmonic functions and involve among others an arbitrary parameter which determines their applicability as charged black holes, dilaton black holes or strings. Most of the known solutions are contained as special cases and can be non-trivially generalized in different ways.
\end{abstract}

PACS No.: 12.10.Gq, 04.20.Jb 


\section{Introduction}

The study of the gravitational interaction coupled to the Maxwell and dilaton fields has been the subject of recent investigations. Einstein-Maxwell fields of black hole type are probably the most interesting objects predicted by classical general relativity. Dilaton fields appear (coupled to Einstein-Maxwell fields) naturally in the low-energy limit of string theory and as a result of a dimensional reduction of the Kaluza-Klein Lagrangian. Therefore, the study of the dilaton field coupled to matter is of importance for the understanding of more general theories. In this work we investigate the Lagrangian density [1]

$$
\mathcal{L}=\sqrt{-g}\left[-R+2(\Delta \Phi)^{2}+e^{-2 \alpha \Phi} F^{2}\right]
$$

where $g=\operatorname{det}\left(g_{\mu \nu}\right), \mu, \nu=0,1,2,3, R$ is the scalar curvature, $F_{\mu \nu}$ is the Maxwell field, and $\Phi$ is the dilaton. The constant $\alpha \geq 0$ determines the special theories contained in Eq. (四). For $\alpha=\sqrt{3}$, the Lagrangian (1) leads to the Kaluza-Klein field equations obtained from the dimensional reduction of the five-dimensional Einstein vacuum equations. For $\alpha=1$, the Lagrangian concides with the low energy limit of string theory with vanishing dilaton potential [2]. Finally, in the extreme limit $\alpha=0$, Eq. (11) reduces to the Einstein-Maxwell theory minimally coupled to the scalar field.

The field equations obtained from Eq. (1) are

$$
\begin{aligned}
\left(e^{-2 \alpha \Phi} F^{\mu \nu}\right)_{; \mu} & =0 \\
\Phi_{; \mu}^{; \mu}+\frac{\alpha}{2} e^{-2 \alpha \Phi} F_{\mu \nu} F^{\mu \nu} & =0 \\
R_{\mu \nu}=2 \Phi_{, \mu} \Phi_{, \nu} & +2 e^{-2 \alpha \Phi}\left(F_{\mu \lambda} F_{\nu}{ }^{\lambda}-\frac{1}{4} g_{\mu \nu} F_{\alpha \beta} F^{\alpha \beta}\right),
\end{aligned}
$$

where a semicolon denotes the covariant derivative with respect to $g_{\mu \nu}$ and a comma represents partial differentiation. A few exact solutions of Eqs.(2- 4) are known which reveals many interesting features of the dilaton field (see ref. [1] and the literature cited therein). In this paper, we obtain several solutions to Eqs.(2- (4) by applying the potential space formalism originally developed by Neugebauer and Kramer for Einstein-Maxwell fields [3].

We assume that the spacetime is characterized by two Killing vector fields, $X$ and $Y$, and introduce coordinates $t$ and $\phi$ which are chosen as $X=\partial / \partial t$ and $Y=\partial / \partial \phi$. The corresponding line element can then be expressed as

$$
d s^{2}=f(d t-\omega d \phi)^{2}-f^{-1}\left[e^{2 k}\left(d \rho^{2}+d z^{2}\right)+\rho^{2} d \phi^{2}\right],
$$

where $f, \omega$ and $k$ are functions of $\rho$ and $z$ only.

In section 2 we introduce the abstract potential space that leads to a set of equations

equivalent to the field equations (2-4) for the metric (5). It turns out that for special values 
of the parameter $\alpha$, the main field equations may be reduced to a chiral equation which can be solved by using the method of harmonic maps. In section 3 we present two classes of exact solutions the first of which describes a general static gravitational field coupled to a dilaton field, both of them being determined by an arbitrary harmonic function. The second class of solutions contains two harmonic functions which determine the gravitational, dilaton and electric fields. Several particular solutions of these classes are derived and briefly discussed.

\section{The Potential Space}

Due to the non-minimal coupling of the electromagnetic and dilaton fields in the Lagrangian (1), the explicit form of the field equations for the line element (5) becomes very cumbersome. It turns out that the metric functions $f$ and $\omega$ must satisfy two partial, second order differential equations which depend also on the electromagnetic and dilaton fields. Moreover, the function $k$ satisfies two partial, first order differential which, however, can be integrated by quadratures once $f$, and $\omega$ are known. In general, it is very hard to obtain directly exact solutions to this system. Therefore, we will apply here a simplifying approach which is based upon the introduction of an abstract space with coordinates defined by the metric functions entering (5). To obtain the "metric" that determines the abstract space, we introduce the line element (5) into the Lagrangian (11) and neglect the total divergence terms which contain second order derivatives. The resulting Lagrangian depends on the metric functions as well as their first order derivatives, and may be handle like a Lagrangian for a mechanical system with coordinates which coincide with the metric functions of (5). We then apply a Legendre transformation involving all the cyclic coordinates of the Lagrangian. This procedure is similar to that used in classical mechanics to obtain the Routh function, and has recently been used in other cases [4], [5]. The resulting Lagrangian is then the metric determining the abstract space, and may be written as

$$
\mathcal{L}=d S^{2}=\frac{\rho}{2 f^{2}}\left[D f^{2}+(D \epsilon+\psi D \chi)^{2}\right]+\frac{\rho \kappa^{2}}{2 f}\left(D \psi^{2}+\frac{1}{\kappa^{4}} D \chi^{2}\right)+\frac{2 \rho}{\alpha^{2} \kappa^{2}} D \kappa^{2}
$$

where $D=(\partial / \partial \rho, \partial / \partial z)$ is a vector operator with its "dual" $\tilde{D}=(\partial / \partial z,-\partial / \partial \rho)$ such that $D \tilde{D} G(\rho, z)=0$ for any arbitrary function $G(\rho, z)$. The "coordinates" of (6) are defined by the following equations

$$
\psi=2 A_{t}, \quad \kappa^{2}=e^{-2 \alpha \Phi}, \quad \tilde{D} \chi=2 \frac{f \kappa^{2}}{\rho}\left(\omega D A_{t}+D A_{\phi}\right), \quad \tilde{D} \epsilon=\frac{f^{2}}{\rho} D \omega+\psi \tilde{D} \chi,
$$

where the electromagnetic vector potential $A_{\mu}=\left(A_{t}, 0,0, A_{\phi}\right)$ has two non-vanishing components only, in accordance to the symmetry properties of the gravitational field. According to their definition, the coordinates $f, \epsilon, \psi, \chi$ and $\kappa$ entering Eq. (6) may also be interpreted 
as the gravitational, rotational, electric, magnetic, and scalar potentials, respectively. The variation of (6) with respect to the potentials leads to the Klein-Gordon equation

$$
D^{2} \kappa+\frac{1}{\rho} D \rho D \kappa-\frac{1}{\kappa} D \kappa^{2}-\frac{\alpha^{2}}{4 f}\left(\kappa^{3} D \psi^{2}-\frac{1}{\kappa} D \chi^{2}\right)=0,
$$

the Maxwell equations

$$
\begin{aligned}
& D^{2} \psi+\left(\frac{D \rho}{\rho}+2 \frac{D \kappa}{\kappa}-\frac{D f}{f}\right) D \psi-\frac{1}{\kappa^{2} f}(D \epsilon+\psi D \chi) D \chi=0, \\
& D^{2} \chi+\left(\frac{D \rho}{\rho}-2 \frac{D \kappa}{\kappa}-\frac{D f}{f}\right) D \chi+\frac{\kappa^{2}}{f}(D \epsilon+\psi D \chi) D \psi=0,
\end{aligned}
$$

and the Einstein equations

$$
\begin{aligned}
& D^{2} f+\frac{1}{f}\left[(D \epsilon+\psi D \chi)^{2}-D f^{2}\right]+\frac{1}{2 \kappa^{2}}\left(\kappa^{4} D \psi^{2}+D \chi^{2}\right)=0 \\
& D^{2} \epsilon+D \psi D \chi+\psi D^{2} \chi+(D \epsilon+\psi D \chi)\left(\frac{D \rho}{\rho}-2 \frac{D f}{f}\right)=0 .
\end{aligned}
$$

Notice that the latter system of differential equations and the Lagrangian (6) do not contain the function $k$ explicitly. This is due to the fact that $k$ is a cyclic coordinate of the original Lagrangian (1) and, as pointed above, a Legendre transformation has been used which eliminates all the terms containing the respective "velocity" $D k$ from the Lagrangian (6). As mentioned above, from the original field equations we obtain two differential equations for $k$ that may be written in terms of the coordinates of the abstract space in the following form

$$
\begin{aligned}
k_{\rho}= & \frac{\rho}{4 f^{2}}\left[f_{\rho}^{2}-f_{z}^{2}+\epsilon_{\rho}^{2}-\epsilon_{z}^{2}+\left(\frac{f}{\kappa^{2}}+\psi^{2}\right)\left(\chi_{\rho}^{2}-\chi_{z}^{2}\right)\right. \\
& \left.-2 \psi\left(\epsilon_{\rho} \chi_{\rho}-\epsilon_{z} \chi_{z}\right)+\kappa^{2} f\left(\psi_{\rho}^{2}-\psi_{z}^{2}\right)+\left(\frac{2 f}{\alpha \kappa}\right)^{2}\left(\kappa_{\rho}^{2}-\kappa_{z}^{2}\right)\right] \\
k_{z}= & \frac{\rho}{2 f^{2}}\left[f_{\rho} f_{z}+\epsilon_{\rho} \epsilon_{z}+f \psi_{\rho} \psi_{z}-\psi\left(\epsilon_{\rho} \chi_{z}+\epsilon_{z} \chi_{\rho}\right)+\left(\frac{f}{\kappa^{2}}+\psi^{2}\right) \chi_{\rho} \chi_{z}+\left(\frac{2 f}{\alpha \kappa}\right)^{2} \kappa_{\rho} \kappa_{z}\right] .
\end{aligned}
$$

Since Eqs. (8-12) follow from the Euler-Lagrange equation applied to the Lagrangian (6) and the latter can be considered as a line element in the potential space, then we conclude that any solution to the field equations (6) (8) may be interpreted as a geodesic in a fivedimensional abstract space with "coordinates" $f, \epsilon, \psi, \chi$ and $\kappa$. This interpretation is of special interest since one can use the symmetries of the geodesics generated by (fivedimensional) Killing vectors, affine collineations, etc. in order to relate different solutions in the (four-dimensional) spacetime. However, this task is beyond the scope of this work and will be treated in further investigations. 


\section{Exact Solutions}

It is still very difficult to find exact solutions to the system of coupled differential equations (\$12). In a recent work [6], Matos investigated a special case $(\alpha=\sqrt{3})$ of the Lagrangian (11) in five dimensions and found out that the solution of the main field equations can be reduced to an equivalent linear problem, based on the Lax pair representation, which leads to a chiral equation for certain combinations of the spacetime metric functions or, equivalently, of the potentials in the abstract space. The respective chiral equation turns out to be invariant with respect to $S L(3, R)$-transformations and hence solutions of the field equations can be classified with respect to the subgroups of $S L(3, R)$. It can be shown that the existence of a chiral equation is related to the geometric properties of the potential space. In fact, the reduction to the problem to a chiral equation is allowed only if the potential space corresponds to a Riemannian symmetric space (vanishing of the covariant derivative of the Riemann curvature tensor).

Unfortunately, it is not possible to adapt the results of [6] in the present case because the Riemannian space defined by Eq. (6) is not symmetric and, consequently, no chiral equation exists that could be used to simplify the field equations (8-12). It turns out that this property holds only in the special cases $\alpha=0, \sqrt{3}$. However, we were able to generalize some of the special solutions presented in [6] to include the case of arbitrary $\alpha$. Without details of calculations we present the resulting solutions and briefly comment their properties.

First, we present the general static axisymmetric solution with an arbitrary harmonic dilaton field. The line element is that of Eq. (5) and the potentials are given as

$$
f=e^{\gamma \lambda}, \quad \kappa^{2}=\kappa_{0} e^{\beta \lambda}, \quad \epsilon=\psi=\chi=0,
$$

where $\kappa_{0}$ is an arbitrary constant, $\beta$ and $\gamma$ are constants related by

$$
\beta=\frac{2 \alpha^{2}}{1+\alpha^{2}}, \quad \gamma=\frac{2}{1+\alpha^{2}}
$$

with $\alpha$ being the arbitrary parameter entering the Lagrangian (1). Furthermore, $\lambda=\lambda(\rho, z)$ is a harmonic function, i.e. it satisfies the two-dimensional Laplace equation

$$
\lambda_{, \rho \rho}+\frac{1}{\rho} \lambda_{, \rho}+\lambda_{, z z}=0 .
$$

According to Eqs. (13) and (14), the metric function $k$ can be calculated for any given $\lambda$ by means of

$$
k_{, \rho}=\frac{\gamma}{2} \rho\left(\lambda_{, \rho}^{2}-\lambda_{, z}^{2}\right), \quad k_{, z}=\gamma \rho \lambda_{, \rho} \lambda_{, z} .
$$

Equation (15) shows that the harmonic function $\lambda$ determines the gravitational as well as the dilaton field. In the limiting case $b=0(\Phi=0)$, the solution reduces to the wellknown Weyl static vacuum solution [7]. For an asymptotically flat spacetime, the function 
$\lambda$ may be chosen as

$$
\lambda=\sum_{n=0}^{\infty} q_{n} \frac{P_{n}(\cos \theta)}{\left(\rho^{2}+z^{2}\right)^{\frac{n+1}{2}}}, \quad \cos \theta=\frac{z}{\left(\rho^{2}+z^{2}\right)^{1 / 2}},
$$

where $q_{n}$ are arbitrary constants and $P_{n}(\cos \theta)$ are the Legendre polynomials of order $n$. A special case of the general solution is that of a Schwarzschild-like black hole dilaton which corresponds to the choice $\lambda=\lambda_{S}=\ln (1-2 m / r)$, where $m=$ const. and $r$ is a radial coordinate determined by $\rho=\sqrt{r^{2}-2 m r} \sin \theta$ and $z=(r-m) \cos \theta$. This solution was first obtained by Janis, Newman and Winicour [8]. They analyzed the behavior of the Schwarzschild sphere $r=2 m$, showed that it becomes a singular point, and conjectured that the truncated Schwarzschild solution, where the space suddenly collapses from a radius slightly greater than $r=2 m$ to zero, is a more likely final state of a generic collapse. This conjecture was further studied by Christodoulou [9], but as no more independent solutions to the EinsteinKlein-Gordon field equations are known, the question remains open. Recently, several time dependent solutions to these field equations has been found, se for example Ref. [10]. This fact, together with the general solution for an arbitrary harmonic dilaton presented in this work, will contribute to settle down the question of the final state of a generic collapse as well as that about the cosmic censorship conjecture. For instance, the choice $\lambda=\lambda_{S}+\lambda_{M}$, where $\lambda_{M}$ is an arbitrary harmonic function may be used to add a multipole structure to the Schwarzschild-like dilaton, a solution that could be used to describe the initial state of a collapsing configuration.

The generic form of the second class of solutions may be written as

$$
\begin{array}{r}
f=f_{0} \frac{e^{\lambda_{0} \lambda+\tau_{0} \tau}}{\left(a_{1} \Sigma_{1}+a_{2} \Sigma_{2}\right)^{\gamma}}, \quad \kappa^{2}=\kappa_{0}^{2}\left(a_{1} \Sigma_{1}+a_{2} \Sigma_{2}\right)^{\beta} e^{\lambda_{0} \lambda+\left(\tau_{0}-q_{1}-q_{2}\right) \tau}, \\
\psi=\frac{a_{3} \Sigma_{1}+a_{4} \Sigma_{2}}{a_{1} \Sigma_{1}+a_{2} \Sigma_{2}}, \quad \epsilon=\chi=0,
\end{array}
$$

where $a_{1}, \ldots, f_{0}, q_{1}, q_{2}, \kappa_{0}, \lambda_{0}$, and $\tau_{0}$ are constants. Furthermore, $\lambda=\lambda(\rho, z)$ and $\tau=\tau(\rho, z)$ are harmonic functions; $\Sigma_{1}$ and $\Sigma_{2}$ represent functions that can be given in terms of $\tau$. In fact, Eq. (20) contains two further subclasses that follow from the different values of the functions $\Sigma_{1}$ and $\Sigma_{2}$. The first one corresponds to the choice

$$
\Sigma_{1}=e^{q_{1} \tau}, \quad \Sigma_{2}=e^{q_{2} \tau} .
$$

In this case, the constants entering the solution (20) are related by

$$
4 a_{1} a_{2} f_{0}+\kappa_{0}^{2}\left(1+\alpha^{2}\right)\left(a_{1} a_{4}-a_{2} a_{3}\right)^{2}=0 .
$$

The electric potential $\psi$ is completely determined by the harmonic function $\tau$ which always appears in the exponential function. To obtain the second subclass of solution (20), we must 
restrict the values of the constants $q_{1}$ and $q_{2}$, and specify $\Sigma_{1}$ and $\Sigma_{2}$ as follows

$$
q_{1}=-q_{2}, \quad \text { and } \quad \Sigma_{1}=\tau, \quad \Sigma_{2}=1 .
$$

In contrast to subclass (22), the electric potential $\psi$ is now given as the ratio of two linear combinations of $\tau$. For the choice (23), the constants satisfy the relationship

$$
4 a_{1}^{2} f_{0}-\kappa_{0}^{2}\left(1+\alpha^{2}\right)\left(a_{1} a_{4}-a_{2} a_{3}\right)^{2}=0 .
$$

The generic solution (20) contains two arbitrary harmonic functions and a large number of constants. This allows us to generate particular solutions with totally different properties. The electric potential $\psi$ can be made to correspond to any desired electric multipole structure (monopole, dipole, quadrupole, etc.) by appropriately choosing the value of the harmonic function $\tau$. Once $\tau$ has been fixed, it still remains the freedom of the function $\lambda$ which can be used to fix the gravitational potential $f$ such that it describes an arbitrary mass multipole configuration. Consequently, the harmonic functions $\lambda$ and $\tau$ allow us to "construct" any arbitrary combination of mass and electric multipoles. So far only electrically charge solutions may be derived from the generic solution (20). However, it is easy to see that the field equations (2-4) are invariant under the transformation

$$
\Phi \rightarrow-\Phi, \quad F_{\mu \nu} \rightarrow F_{\mu \nu}^{*}=\frac{1}{2} e^{-2 \alpha \Phi} \eta_{\mu \nu \rho \sigma} F^{\rho \sigma}
$$

where $\eta_{\mu \nu \rho \sigma}$ is the Levi-Civita pseudotensor. This duality rotation, which also involves the dilaton field, may be used to generate the magnetic counterpart of the generic class (20). Accordingly, for any particular electrically charged solution contained in (20), the magnetically charged solution may be obtained by changing the sign of the dilaton.

Now we derive some special solutions contained in (20). Consider the subclass (23) with the following special values of the constants

$$
\tau_{0}=\lambda_{0}=a_{3}=0, \quad a_{1}=a_{2}=f_{0}=\alpha=1=\kappa_{0}^{2}=1 .
$$

According to Eqs. (23), the resulting solution takes the form

$$
f=\frac{1}{1+\tau}, \quad \kappa^{2}=1+\tau, \quad \psi=\frac{\sqrt{2}}{1+\tau},
$$

which is equivalent to the static dilatonic version of the Kastor-Trashen [11], [2] solution for the value $\tau=\sum 2 M_{i} /\left|r-r_{i}\right|$. Here $M_{i}$ and $r_{i}$ are positive constants, the sum is over all positive integer values of $i$, and $r$ represents a radial coordinate. This solution represents a collection of extremal electrically charged black holes which are static because the electric repulsion is balanced by both the gravitational and dilatonic attraction. Originally, this 
solution was derived in the low energy limit of string theory $(\alpha=1)$, but letting in (23) $\alpha$ arbitrary we easily obtain the generalization to Kaluza-Klein and Einstein-Maxwell theories: $f=(1+\tau)^{-\gamma}, \kappa^{2}=(1+\tau)^{\beta}, \psi=2\left(1+\alpha^{2}\right)^{-1 / 2}(1+\tau)^{-1}$, where $\gamma$ and $\beta$ are given as in Eq. (16). This new exact solution could be important in the understanding of the event horizon, since constant $\alpha$ obviously adds new structure to the horizon of the static case of the Kastor-Trashen solution.

Another interesting special solution may be obtained by considering the subclass (21) with the following values of the constants

$$
f_{0}=\kappa_{0}^{2}=1, \quad q_{1}=\lambda_{0}=0, \quad \tau_{0}=q_{2}, \quad a_{1}=1-a_{2}=\frac{r_{+}}{r_{+}-r_{-}}, \quad a_{4}=-a_{3}=\frac{2 Q}{r_{+}-r_{-}},
$$

where $r_{+}, r_{-}$, and $Q$ are constants. With this choice of the constants the harmonic function $\lambda$ does not enter the resulting solution. We fix the remaining function $\tau$ as

$$
\tau=\frac{1}{q_{2}} \ln \left(1+\frac{r_{-}-r_{+}}{r}\right)
$$

where $r$ is a radial coordinate related to cylindrical coordinates by means of $\rho=$ $\sqrt{r^{2}+\left(r_{-}-r_{+}\right) r} \sin \theta$ and $z=\left[r+\left(r_{-}-r_{+}\right) / 2\right] \cos \theta$. Inserting these values into Eqs. (20]) and 21, we obtain

$$
f=\left(1+\frac{r_{-}-r_{+}}{r}\right)\left(1+\frac{r_{-}}{r}\right)^{-\gamma}, \quad \kappa^{2}=\left(1+\frac{r_{-}}{r}\right)^{\beta}, \psi=-\frac{2 Q}{r+r_{-}}
$$

This solution was originally obtained by Gibbons and Maeda [12]; the representation given here coincides with that of Horne and Horowitz [1] (after performing the coordinate displacement $r \rightarrow r+r_{-}$in Eqs. (6-10) of Ref. [1]).

Another special solution contained in (20) was recently given by Matos [13 for an arbitrary magnetic field coupled to the dilaton. It is direct to see that it is contained in (20) by using the dual transformation given in Eq. (25), and setting $\tau_{0}=0$ and $\alpha=\sqrt{3}$.

All the special solutions given here contain other particular solutions in each of theories that can be obtained by specifying the value of $\alpha$. We believe that all known static solutions of the field equations following from the Lagrangian (1) are contained in Eqs. (20), (21) and (23) as special cases. It would be interesting to find stationary solutions with both electric and magnetic field. This could be done by using the symmetry properties of the metric (6) of the potential as mentioned above. In fact, we obtained all the Killing vectors of metric (6) but, unfortunately, they cannot be used to generate solutions since they correspond to "gauge transformations" of the potential, i.e., the "stationary electromagnetic" solutions generated by applying infinitesimal Killing transformations on the solution (20) contain only trivial angular momentum and magnetic fiel terms that can be eliminated by appropriate coordinate 
transformations. Consequently, we have to derive more general symmetry properties of the potential space (affine collineations, curvature collineations, etc.). This task will be treated in further investigations.

\section{References}

[1] J.H. Horne and G.T. Horowitz, Phys. Rev. D 46, 1340 (1992).

[2] J.H. Horne and G.T. Horowitz, Phys. Rev. D 48, R5457 (1993).

[3] G. Neugebauer and D. Kramer, Ann. Phys. (Leipzig) 24, 62 (1969).

[4] D. Núñez and H. Quevedo, SILARG VIII. Proceedings of the $8^{\text {th }}$ LAtin American Symposium on Relativity and Gravitation, (Águas de Lindóia, Brazil, 1993), Eds. P. S. Letelier and W. A. Rodriguez, Jr, World Scientific, p. 162, 1994.

[5] D. Núñez and H. Quevedo, submitted to Journ. Math. Phys. (1993).

[6] T. Matos, Journ. Math, Phys. 351302 (1994).

[7] D. Kramer, H. Stephani, E. Herlt, and M. MacCallum, Exact Solutions of Einstein's Field Equations, Cambridge University Press, Cambridge (1980).

[8] A.J. Janis, E.T. Newman and J. Winicour, Phys. Rev. Lett. 20, 878 (1968).

[9] D. Chistodolou, Comm. Math. Phys. 105, 337 (1986); 106, 587 (1986); 109, 591 (1987); 109, 613 (1987).

[10] V. Husain, E. A. Martinez, D. Núñez, Phys. Rev. D (1994), in press.

[11] D. Kastor and J. Trashen, Phys. Rev. D 47, 5370 (1993); J.H. Horne and G.T. Horowitz, Phys. Rev. D 48, R5457 (1993).

[12] G. Gibbons and K. Maeda, Nucl. Phys. B 298, 741 (1988).

[13] T. Matos, Phys. Rev. D 49, 4296 (1994). 